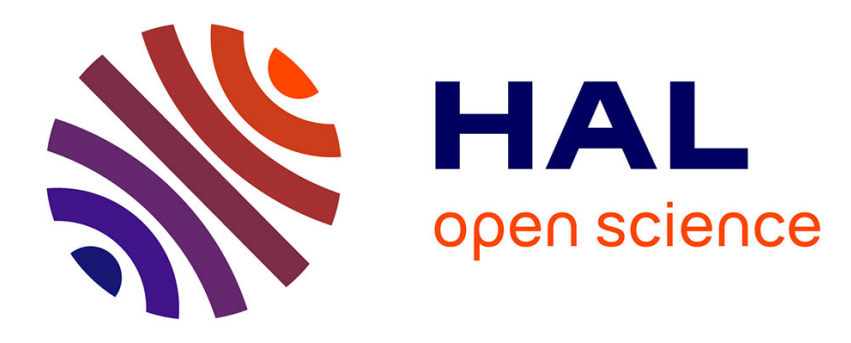

\title{
Les tiers de procréation dans les familles homoparentales Martine Gross
}

\section{- To cite this version:}

Martine Gross. Les tiers de procréation dans les familles homoparentales. Recherches familiales, 2014, 11, pp.19-30. hal-00993204

\section{HAL Id: hal-00993204 https://hal.science/hal-00993204}

Submitted on 20 May 2014

HAL is a multi-disciplinary open access archive for the deposit and dissemination of scientific research documents, whether they are published or not. The documents may come from teaching and research institutions in France or abroad, or from public or private research centers.
L'archive ouverte pluridisciplinaire HAL, est destinée au dépôt et à la diffusion de documents scientifiques de niveau recherche, publiés ou non, émanant des établissements d'enseignement et de recherche français ou étrangers, des laboratoires publics ou privés. 


\title{
LES TIERS DE PROCRÉATION DANS LES FAMILLES HOMOPARENTALES
}

\author{
Martine Gross
}

Dans cet article, nous explorons les représentations de la parenté vis-à-vis du tiers de procréation à l'œuvre chez les hommes et les femmes qui fondent une famille homoparentale. Après un bref état des lieux de la diversité des configurations homoparentales, nous nous pencherons sur deux questions. La première est celle des termes d'adresse et de désignation des liens. Dans la mesure où, jusqu'en 2013, il n'était pas envisageable d'être deux parents de même sexe du point de vue du droit, comment se désigne-t-on et comment se fait-on appeler quand on n'est pas un parent statutaire? La deuxième concerne les termes utilisés quand on parle des personnes, donneur ou donneuse de gamètes, et/ou gestatrice, les tiers de procréation qui contribuent à donner la vie à un enfant sans en être un parent. Nous examinerons l'articulation entre les discours tenus à propos des tiers de procréation et les termes d'adresse ou de désignation du lien. Pour conclure, nous nous interrogerons sur les manières différentes qu'ont les hommes et les femmes de désigner ou de considérer les tiers de procréation. En considérant l'implication corporelle des donneurs de sperme, des donneuses d'ovocyte et des femmes gestatrices, nous mettrons en regard l'articulation des liens biologiques et sociaux chez les couples hétérosexuels infertiles et les couples de même sexe.

Nous nous intéresserons dans cet article aux couples de femmes et aux couples d'hommes qui ont choisi de fonder une famille homoparentale en recourant à un tiers pour procréer. Pour les femmes, ce tiers peut être un donneur anonyme ou semi-anonyme ${ }^{[1]}$ dans le cadre d'une procréation médicalement assistée avec don de sperme. Il peut s'agir d'un ami qui accepte de contribuer à la conception d'un enfant et de ne pas revendiquer sa paternité, c'est ce que nous désignerons dans la suite de l'article par l'expression « donneur connu ». Il peut s'agir d'un père ou de deux pères ${ }^{[2]}$ dans le cadre d'une coparentalité. La coparentalité en contexte homoparental est une situation dans laquelle un homme et une femme sans vie conjugale commune, généralement tous deux homosexuels, conçoivent et élèvent leurs enfants au sein de leurs deux foyers : le foyer maternel et le foyer paternel. Il peut y avoir de 2 à 4 personnes impliquées dans le projet parental si la compagne de la mère ou le compagnon du père sont parties prenantes du projet. Les hommes de leur côté doivent, pour procréer, recourir à une femme qui portera leur enfant. Il peut s'agir d'une gestatrice et d'une donneuse d'ovocyte s'ils ont recours à une gestation pour autrui ou bien une mère ou deux mères dans le cadre d'une coparentalité. La procréation médicalement assistée étant réservée depuis les lois de bioéthique de 1994 aux couples

[1] Lors du don semi-anonyme, le donneur consent à ce que son identité soit transmise à l'enfant si celui-ci le demande à sa majorité. Le don semi-anonyme est pratiqué aux Pays-Bas. Certaines cliniques de fertilité en Belgique le proposent.

[2] J'utilise le terme de père ou de mère lorsque la personne interrogée se vit et dit s'impliquer comme tel. 
hétérosexuels souffrant d'une pathologie de la fertilité, les couples de femmes se rendent en Belgique ou en Espagne pour recourir à une insémination artificielle avec don de sperme. La gestation pour autrui est interdite en France, les hommes qui ont recours à cette pratique se rendent à l'étranger dans les pays où elle est légale. L'ouverture du mariage et de l'adoption aux couples de même sexe n'ouvre pas la procréation médicalement assistée aux couples de femmes et n'a pas envisagé de légaliser le recours à la GPA. Jusqu'au vote de la loi en avril 2013, un enfant né dans un contexte homoparental ne pouvait avoir deux parents de même sexe. La nouvelle loi permet d'établir la filiation d'un enfant envers la compagne de la mère ou le compagnon du père en passant par le mariage et l'adoption par le conjoint ou la conjointe. Nous explorerons, en nous appuyant sur plusieurs enquêtes menées auprès d'hommes et de femmes qui ont choisi de recourir à un ou des tiers pour procréer, les représentations qu'ils et elles se font de la parenté en examinant la désignation des liens à l'enfant, les termes d'adresse et le discours tenus sur les tiers de procréation. Ces indicateurs permettent d'analyser les discours tenus et ne disent rien des pratiques parentales au quotidien ${ }^{[3]}$.

\section{$\checkmark$ Foyers lesbiens}

Trois enquêtes par questionnaire auprès des adhérents de l'Association des Parents et futurs parents Gays et Lesbiens (APGL) ont été réalisées entre 1997 et 2005. La première en 1997, la deuxième en 2001 et la troisième en $2005^{[4]}$. Les données issues de ces enquêtes ne prétendent pas représenter l'ensemble des familles homoparentales en France. Obtenir un échantillon représentatif est impossible, faute de connaitre la population de référence. Ces enquêtes ont permis d'explorer, dans le cadre de cette association, les modes de désignation de la compagne de la mère légale, les termes employés par les enfants pour s'adresser à elle ainsi que ceux utilisés pour évoquer l'homme qui a contribué à donner la vie ${ }^{[5]}$.

\section{$\checkmark$ Termes d'adresse}

Entre 1997 et 2005, la manière dont les enfants s'adressent à la compagne de leur mère légale ou sont encouragés à le faire a évoluée ${ }^{[6]}$. Le terme de parenté spirituelle «marraine », encore utilisé dans les années 1990, disparaît dans la période plus récente. La plupart des compagnes se font appeler par un petit nom maternel ${ }^{[7]}$ : « mamou », « mamina », ou par un mot qui signifie maman dans une autre langue. L'appellation par le prénom est de moins en moins fréquente, tandis que « maman » seul ou suivi du prénom connaît une progression certaine.

[3] La littérature scientifique en France ne manque pas sur les familles homoparentales. L'anthropologue Anne CADORET a décrit la diversité des figures homoparentales (Des parents comme les autres, Odile Jacob, 2002). Pour une étude des pratiques parentales des couples de femmes qui, confrontées à l'hétéronormativité de l'environnement, ont construit ensemble leur projet parental, on se référera aux travaux de Virginie DESCOUTURES (Les mères lesbiennes, PUF, 2010). Ceux d'Emmanuel GRATTON (L'homoparentalité au masculin, PUF, 2008) ainsi que ma récente enquête sur la paternité gay (Choisir la paternité gay, Érès, 2012) permettent d'aborder le désir d'enfant chez les pères gays et les caractéristiques de leurs projets parentaux.

[4] Ces enquêtes ont été complétées en 2005 par quelques entretiens d'adhérentes qui avaient laissé leurs coordonnées lors de la remise du questionnaire. Par ailleurs, durant l'automne 2006, une discussion animée a eu lieu de manière spontanée sur la mailing liste de l'Association des parents gays et lesbiens (APGL) à propos du nom à employer pour désigner la compagne de la mère légale. Nous avons obtenu l'autorisation d'utiliser ces témoignages dans le cadre de ce travail.

[5] Martine GROSS, «Deux parents ou deux mamans ?Évolution des termes d'adresse et de désignation dans les familles lesboparentales », in Didier LE GALL (dir.), Identités et Genres de vie. Chroniques d'une autre France, Paris, L'Harmattan, 2008, pp. 199-216.

[6] Ibid.

[7] La modalité « petit nom » n'était pas proposée en 1997 et est donc absente dans le traitement statistique pour cette date. 


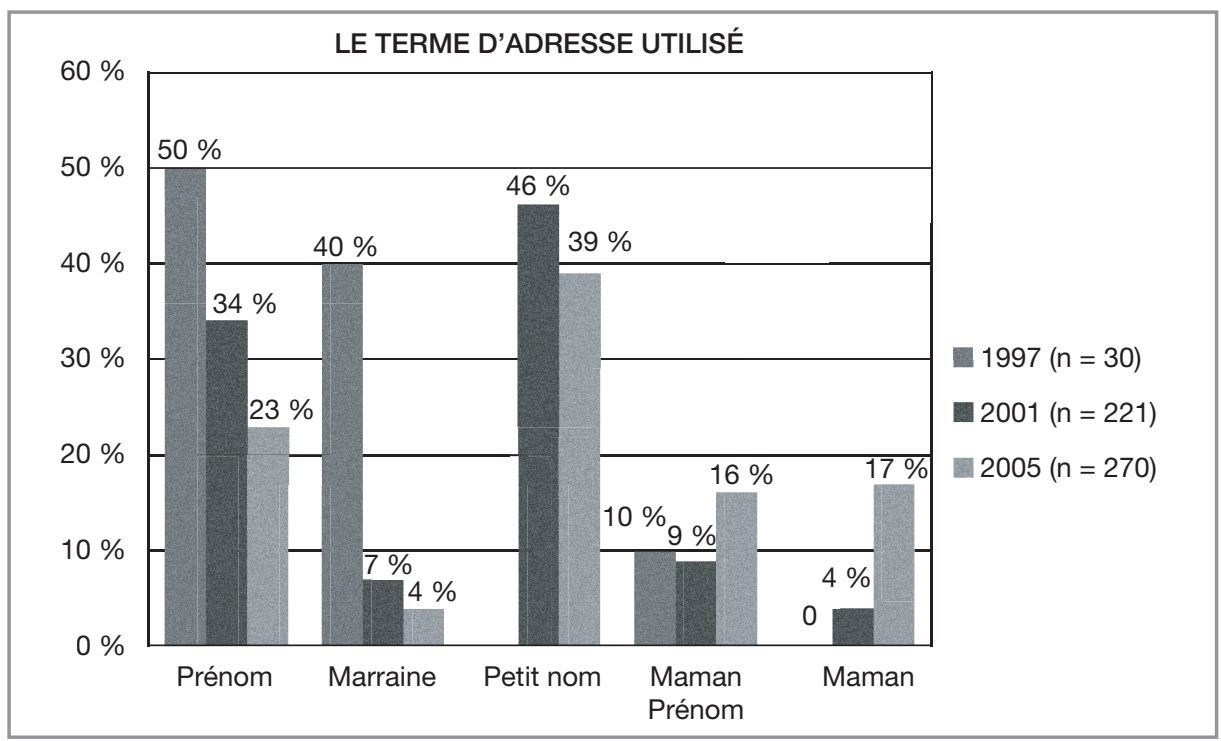

\begin{tabular}{|l|c|c|c|}
\hline & $1997(n=30)$ & $2001(n=221)$ & $2005(n=270)$ \\
\hline Prénom & $50 \%$ & $34 \%$ & $23 \%$ \\
\hline Marraine & $40 \%$ & $7 \%$ & $4 \%$ \\
\hline Petit nom & & $46 \%$ & $39 \%$ \\
\hline Maman Prénom & $10 \%$ & $9 \%$ & $16 \%$ \\
\hline Maman & 0 & $4 \%$ & $17 \%$ \\
\hline
\end{tabular}

Il faut noter que dans cette période, les modalités choisies pour fonder une famille homoparentale ont également évolué. Davantage de femmes ont opté pour l'insémination artificielle, délaissant la coparentalité, avec un discours soulignant la nature conjugale de leur projet parental. Il en résulte un changement dans la manière dont les compagnes des mères légales tendent à se situer désormais dans ce projet d'enfant. Ce que confirment les réponses des compagnes des mères légales à cette autre question : «Comment vous situez-vous dans le projet parental ? ». La réponse « Comme un futur parent moi-même », qui était déjà largement plébiscitée en 2001, continue de croitre, aux dépens de «Comme associée au projet mais sans être parent moi-même».

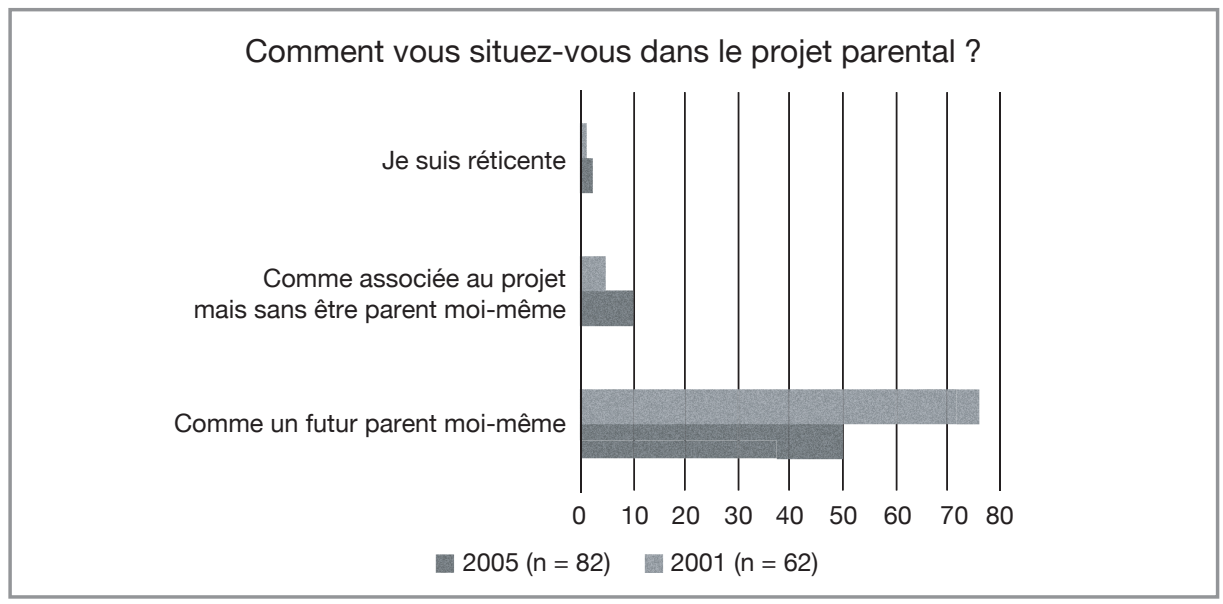


Autrement dit, au sein des foyers lesbiens enquêtés, qui recourent de plus en plus à l'insémination artificielle avec don de sperme (IAD) pour se constituer, non seulement le projet d'avoir un enfant est bel et bien un projet de couple, mais les compagnes des mères légales disent de plus en plus s'investir dans ce projet comme un parent à part entière ${ }^{[8]}$.

\section{$\checkmark$ Désignation des liens}

Dans l'analyse de la désignation des liens dans les foyers lesbiens, je m'étais intéressée au caractère sexué ou neutre des mots employés (parent $v s$ mère) dans la réponse à la question « comment racontez-vous sa conception ou son histoire à votre enfant ? ». Déclarer : « nous sommes ses deux mamans » n'est en effet pas strictement équivalent au fait de dire : «nous sommes ses deux parents ». Contrairement à « deux mamans », l'expression « deux parents » n'est pas sexuée. Utiliser l'expression « deux parents » représente un compromis avec la norme. Le couple de femmes peut en effet, en se présentant comme deux parents, se conformer à la norme biparentale sans mettre en cause frontalement la norme de l'altérité sexuelle qui dit « une seule femme/ mère, un seul homme/père ».

Chez les femmes interrogées, le terme neutre de «parents » s'accompagne assez souvent d'une différenciation, d'une individuation de chacune dans la manière de se faire appeler. La neutralité du terme « parent » recouvre une situation où l'enfant est en réalité encouragé à distinguer sa mère statutaire ${ }^{[9]}$ et l'autre. L'enfant s'adresse alors à la compagne avec un «petit nom », voire par son prénom, tandis que celle qui a donné la vie est appelée «maman ».

En revanche, lorsque le discours est sexué, ce qui était le cas pour la plupart des répondantes, comme dans « il a deux mamans », il est nécessairement symétrique. Les enfants s'adressent souvent à la compagne de la mère statutaire par le terme « maman » ou « maman suivi du prénom ».

Dire que l'enfant n'a qu'une seule maman, dire qu'il a « deux parents » plutôt que « deux mères » ou « deux mamans », traduit l'adhésion au modèle traditionnel selon lequel la seule et unique mère est celle qui accouche. Les femmes pour lesquelles le mot « maman » est associé à la maternité biologique préfèrent que l'enfant interpelle leur compagne par un autre terme d'adresse. Dans cette représentation, on peut avoir deux parents, car le mot parent peut désigner les fonctions éducatives et nourricières, mais pas deux mamans, car maman ou mère s'attacherait à un fait biologique.

[8] Les enquêtes menées en 2001 et 2005 montraient une progression de la proportion des répondantes compagnes d'une mère biologique qui se situaient comme des parents elles-mêmes, passant de 81 à $93 \%$, tandis que la proportion diminuait de celles qui se déclaraient réticentes au projet ou associées au projet sans être parents elles-mêmes, passant de 19 à $7 \%$. Dans l'enquête «Fonctionnement familial et conjugal des familles homoparentales » menée en 2012 par Jérôme Courduriès, Ainhoa de Federico et moi-même, 94,4 \% des 354 foyers lesbiens où l'aîné des enfants n'était pas né d'une union antérieure, ont déclaré que leur projet parental était un projet de couple.

[9] J'emploie indifféremment l'adjectif « légal » ou l'adjectif « statutaire » utilisé par Virginie DESCOUTURES, pour qualifier celui des deux dans un couple de parents de même sexe, dont la filiation à l'égard de l'enfant est établie. L'autre est un parent seulement « social ». À l'heure où les enquêtes ont été menées, il ne pouvait y avoir deux parents statutaires (ou légaux) de même sexe. Tout au plus, le parent légal pouvait partager avec le parent social (non statutaire) l'exercice de l'autorité parentale. Depuis la loi « Mariage pour tous », il peut y avoir deux parents statutaires : un parent de naissance et un parent adoptif. 
Si la structure des familles lesboparentales remet en question le modèle traditionnel de la famille fondé sur la vraisemblance de liens biologiques entre parents et enfants, il n'en reste pas moins que les représentations culturelles liées à ce modèle traditionnel y sont néanmoins bien présentes.

\section{L'homme qui a donnéla vie: un père? Un gentil monsieur? Un donneur? Un géniteur?}

L'analyse lexicale des réponses libres à la question « comment racontez-vous l'histoire de sa conception à votre enfant ? » de l'enquête menée en 2005 permet également d'explorer la désignation de l'homme qui a contribué à donner la vie.

Le poids des représentations qui attribuent la paternité au géniteur, même si celui-ci n'a aucun rôle auprès de l'enfant, va se faire sentir dans les discours. Toutefois, ceux-ci diffèrent sensiblement selon qu'il y a ou non un homme connu ou/et impliqué auprès de l'enfant. On se doit ici de prendre en compte les trois situations de recours à un tiers : la situation de coparentalité, le donneur connu et l'insémination artificielle de donneur anonyme ou semi-anonyme ${ }^{[10]}$.

Dans la situation de coparentalité, un homme est le père légal de l'enfant et s'implique au quotidien. Dans les récits de conception, il est désigné comme le papa, le père biologique ou un parent. L'histoire n'évoque jamais deux mamans et fait apparaître une hiérarchie entre les parents statutaires et leur compagne et compagnon. Par exemple : "Un papa et une maman pour la conception et deux parents en plus. " Seule la mère biologique se fait appeler maman par l'enfant, tandis que ce n'est jamais le cas pour sa compagne.

Le donneur connu est généralement un ami, qui a accepté de faire don de son sperme pour qu'un enfant vienne au monde. Il n'a cependant pas le statut légal de père puisqu'il n'a pas reconnu l'enfant et n'a en principe pas l'intention de le faire. Il occupe, selon les cas, une place variable dans le quotidien de l'enfant. Dans la grande majorité des récits, lorsque l'enfant est né du recours à un donneur connu, alors qu'il n'a aucune existence légale, et n'est pas impliqué dans le quotidien de l'enfant, ce dernier est pourtant nommé par un terme de parenté. Il est désigné comme «le papa qui donne des graines », "le donneur qui est l'un des parents » ou, plus simplement, "le père qui nous a aidés ». Deux des trois types de lien qui habitent nos représentations en matière de filiation, le sang, le nom, le quotidien, pour reprendre le titre du livre de Florence Weber ${ }^{[11]}$, sont absents de la situation. Le lien du sang identifié (par définition le donneur connu n'est pas anonyme) semble néanmoins suffire pour que le donneur de gamètes reçoive un qualificatif paternel.

Lorsqu'on se penche sur les termes d'adresse employés dans ces situations où le donneur est connu, on constate que les mères sociales ${ }^{[12]}$ de l'enquête se font appeler par leur « prénom » ou par un « petit nom », et jamais par «maman » ou « maman suivi du prénom ». Dès lors qu'il est connu, le géniteur, qu'il soit juste un donneur connu ou qu'il soit un père impliqué dans une

[10] Même si dans certains cas, notamment aux Pays-Bas, l'enfant pourra accéder s'il le souhaite aux informations identifiantes concernant son donneur, nous considérons que le donneur est inconnu puisqu'il l'est pendant toute la minorité de l'enfant.

[11] Florence WEBER, Le sang, le nom et le quotidien, La Courneuve, Aux lieux d'être, 2005.

[12] Voir note 8. 
coparentalité, se retrouve en position de parent et cela semble interdire à la compagne de la mère légale d'être une deuxième maman ou en tout cas de se faire nommer comme telle. Même si le donneur connu n'a qu'une existence ténue dans la vie de l'enfant ou dans celle du couple, la hiérarchie entre liens biologiques et liens seulement sociaux dans les représentations de ce qui fait un « vrai » parent, reprend ses droits. Il n'y a plus de place pour la compagne en tant que parent au même titre que la mère statutaire.

Dans le cas du recours à l'IAD en Belgique ou en Espagne, le donneur n'est pas connu. À la lumière des récits recueillis, la contribution d'un homme est toujours mentionnée, selon diverses déclinaisons allant du « donneur de graines » au «papa », en passant par « géniteur » et «père biologique ». Lorsque les enfants sont jeunes, les donneurs sont souvent évoqués comme « un gentil monsieur qui a donné ses graines », et quelquefois comme « un papa qu'on ne connaît pas » ou « un père inconnu qui a aidé ou qui a donné les graines » et rarement comme « un donneur », terme pressenti comme étant trop technique.

Ce qui ressort de l'étude de 2005, c'est que dès que le géniteur, qu'il s'agisse du père dans la coparentalité, du donneur connu, ou du donneur anonyme, est désigné avec un terme de parenté, qu'on parle de lui comme d'un père biologique, d'un papa qu'on ne connaît pas, les deux femmes ne se font pas appeler toutes les deux " mamans », et ne se considèrent pas comme deux mères. L'utilisation de termes désignant l'homme qui a contribué à donner la vie, comme un père, même s'il n'exerce aucun rôle paternel au quotidien, signe l'acceptation implicite du modèle reproductif « un père, une mère, pas un de plus » qui interdit de se dire deux mères pour le même enfant.

Deux femmes ou deux hommes qui s'auto-instituent en tant que famille remettent en cause notre système de filiation qui, parce qu'il coïncide avec les lois de la génétique, nous incite à penser qu'il est biologiquement fondé, alors qu'il relève d'un choix culturel. Quoi qu'il en soit, comme l'écrit Françoise-Romaine Ouellette ${ }^{[13]}$, « ce modèle ne véhicule pas seulement l'idée que la filiation est un fait de nature. Il s'accompagne surtout d'une norme, celle de l'exclusivité de la filiation, c'est-à-dire que chaque individu n'est mis en position de fils ou de fille que par rapport à un seul homme ou une seule femme ». En France, depuis l'ordonnance de juillet 2005, la femme qui accouche n'a plus besoin de reconnaître l'enfant qu'elle a mis au monde. La filiation maternelle de l'enfant est automatiquement établie envers la femme dont le nom est mentionné dans l'acte de naissance. En quelque sorte, «l'accouchement fait la mère » ${ }^{[14]}$. Aussi, dans ce modèle, se dire mère quand on n'a pas donné la vie ou quand il y a déjà une mère de naissance s'avère impossible. Se dire père alors qu'un autre a déjà pris la place dans le livret de famille ne se peut pas non plus. Un couple de même sexe qui dit «nous sommes deux mères » ou « nous sommes deux pères » ébranle ce modèle exclusif de la filiation. Mais les homoparents ne sont pas les seuls à ébranler ces deux principes, que sont l'exclusivité de la filiation, « un seul père, une seule mère », d'une part, et la naturalité de la filiation maternelle, d'autre part. En témoignent la difficulté à penser la gestation pour autrui mais aussi le statut à donner aux tiers dans les familles recomposées et la possibilité d'accéder à la demande des enfants nés d'un don de connaitre l'identité de ceux qui ont contribué à leur venue au monde. Se dire mère quand on n'a pas

[13] Françoise-Romaine OUELLETTE, « Les usages contemporains de l'adoption », in Agnès FINE (éd.), Adoptions. Ethnologie des parentés choisies, Paris, Éditions de la Maison des sciences de l'homme, 1998, pp. 153-176.

[14] Dans le cas de l'accouchement sous le secret qui autorise une femme à ne pas être la mère de l'enfant auquel elle a donné la vie, l'accouchement est réputé ne pas avoir eu lieu. 
enfanté, comme le font les compagnes de mères lesbiennes, défie le principe de la naturalité de la filiation maternelle. En effet, seul un homme, dans l'état actuel de notre droit, peut se « déclarer » parent aux côtés d'une mère biologique. L'engagement parental que constitue l'établissement de la filiation par la reconnaissance ou par présomption de parenté dans le mariage n'est pas accessible aux compagnes de mères lesbiennes. Elles ont cependant la possibilité depuis le vote au printemps 2013 de la loi ouvrant le mariage et l'adoption aux couples de même sexe, d'adopter l'enfant de leur conjointe.

\section{Fovers gays}

Voyons maintenant ce qu'il en est des hommes qui ont eu recours à la coparentalité ou à la GPA pour fonder une famille homoparentale. Une enquête qualitative par entretiens menée en 2011 a permis de recueillir les témoignages d'une quarantaine d'hommes qui avaient eu recours à une GPA et d'une dizaine qui s'étaient tournés vers la coparentalité ${ }^{[15]}$. Comme chez les couples de femmes, la dimension conjugale est souvent présente. Cette dimension conjugale des projets parentaux diffère de ce que révélaient les enquêtes menées auprès des adhérents de l'APGL en 1998 et en 2005. Même en couple, ils menaient alors des projets individuels de paternité, ne se désignant comme deux pères qu'exceptionnellement ${ }^{[16]}$.

Élever un enfant en coparentalité avec un couple de femmes ou bien l'élever sans mère au quotidien comme c'est le cas dans la GPA, a-t-il un impact sur la manière de se nommer et de se faire appeler par les enfants? Comment les pères gays parlent-ils de la femme qui a porté leur enfant?

\section{$\checkmark$ La coparentalité}

Les couples d'hommes rencontrés qui ont fondé une famille en coparentalité n'ont pas tous évoqué un projet de couple. Plus souvent, l'un est porteur du projet et son compagnon le soutient. Même lorsque le compagnon du père est partie prenante du projet parental, il ne sera au mieux qu'un troisième ou un quatrième parent. En effet, non seulement père et mère statutaires se partagent l'autorité parentale, mais aussi bien souvent le jeune enfant réside la majeure partie du temps chez les mères, ce qui peut positionner le père et son compagnon en position tierce. Les asymétries de genre réapparaissent dans le champ parental en coparentalité, plaçant en concurrence le foyer maternel et le foyer paternel, mais également les couples de même sexe et la dyade parentale statutaire, cristallisant les tensions issues des attentes et des définitions que les hommes et les femmes se donnent de la paternité et de la maternité. Lorsque les pères placent leur paternité sur le même terrain que celui traditionnellement dévolu aux mères, les soins, et revendiquent de partager à égalité avec elles le temps de l'enfant, ces tensions sont parfois inévitables.

[15] Martine GROSS, Choisir la paternité gay, Érès, 2012 ; Martine GROSS, Dominique MEHL, " Homopaternités et GPA », Enfances, familles, générations, ${ }^{\circ}$ 14, 2011, pp. 95-112.

[16] Martine GROSS, « Désir d'enfant chez les gays et les lesbiennes », Terrain, n 46, 2006, pp. 151-154. 
Les hommes rencontrés qui ont choisi la coparentalité ont tendance à identifier origines biologiques et parenté. Certains justifient leur choix de la coparentalité parce qu'ils désirent inscrire leur enfant dans une lignée biologique, ou bien parce qu'ils veulent pouvoir lui raconter d'où il vient. Yves, célibataire de 45 ans, père de deux enfants en coparentalité, expose : «Je pense qu'un enfant doit avoir un père et une mère reconnus, biologiques, savoir d'où il vient. » Dans la coparentalité, procréation et filiation coïncident. D'où peut-être l'expression, dans le discours de certains des hommes interrogés qui ont choisi cette modalité, d'une confusion entre origines biologiques et parents. Concernant la désignation des liens, de fait, certains couples insistent sur le fait qu'il y a un papa et une maman, qu'il n'y a pas deux papas. L'accent mis sur le duo père/ mère peut fragiliser les couples de même sexe. Parmi les situations rencontrées, les coparentalités non conflictuelles sont menées par des pères célibataires ou dont le projet était individuel plutôt que conjugal et qui acceptaient sans amertume la prévalence maternelle.

\section{$\checkmark$ La gestation pour autrui}

Au sein de l'APGL, au début des années 2000, encore très peu d'hommes se tournaient vers la gestation pour autrui. Les coûts, non seulement économique, mais aussi social de la GPA étaient prohibitifs. Recourir à la GPA, c'est risquer d'être perçu comme contribuant à l'exploitation marchande du corps des femmes, c'est braver une interdiction légale. À cette réprobation sociale éventuelle s'ajoute celle liée au fait de priver un enfant d'une mère quotidiennement impliquée. Lors de son étude, Emmanuel Gratton remarque « les représentations d'un enfant que l'on prive de sa mère, d'une mère qui loue son utérus et qui fait le choix de donner son enfant, sont généralement très culpabilisantes. Ceux qui font ce choix se sont libérés de cette culpabilité et des préjugés ». L'enquête menée en 2011 auprès de pères gays ayant eu recours à la GPA, nous fait découvrir une autre réalité : de plus en plus d'hommes gays de l'APGL ont recours à la gestation pour autrui, vivent leur désir d'enfant comme l'aboutissement de leur couple, se définissent comme deux pères, se font appeler papa et daddy, voire papa tous les deux. Ce qui est nouveau avec la GPA, c'est la nature conjugale d'un projet parental où deux hommes seront pères, l'un en procréant, l'autre sans procréer.

Pouvoir dire «nous », se vivre comme deux pères, même si seul l'un deux endosse la paternité légale, est une motivation forte pour choisir la GPA. Il s'agit de fonder une famille dans le prolongement du couple, même si l'enfant n'est pas le fruit biologique de ce couple. Cette motivation est identique à celle qui anime les lesbiennes se rendant à l'étranger pour recourir à une IAD. Chez les hommes gays, elle arrive simplement plus tardivement que chez les lesbiennes. Cette question du «nous » concerne non seulement la qualification conjugale du projet, mais désigne aussi qui sont les parents. Les plus à l'aise avec la disjonction de la paternité et de la procréation n'hésiteront pas à se dire deux pères, tandis que les autres préfèreront se qualifier de parents. Tous les couples rencontrés à l'exception de deux qui estimaient qu'un enfant ne pouvait avoir qu'un seul père et une seule mère, évoquaient un «nous » parental et disaient qu'ils étaient deux pères. Mais, dire « on est deux pères » n'implique pas systématiquement des appellations symétriques. Certains pères se feront appeler tous les deux papa. D'autres trouveront un petit nom à consonance paternelle pour le second père, tel papou, ou l'équivalent de papa dans une 
autre langue, comme daddy. La diversité de ces appellations traduit la palette de nuances dans les représentations et définitions personnelles des liens de parenté, que se donnent ces hommes. Pierre et Jean-Louis se font appeler papa et papou. Jean-Louis explique ce qui a déterminé leur choix : "Notre psychothérapeute avait dit qu'il fallait préparer deux noms, papa pour l'un et autre chose pour l'autre, pour qu'ils ne nous appellent pas tous les deux du même nom. Nous lui faisions tellement confiance que nous n'avions pas remis ça en question. C'est Pierre qui avait trouvé papou. À la maternelle, c'est génial parce que ça nomme un deuxième parent. Sur la porte de classe, ils disent le papa de Céline, la maman de Sofia [...] et moi, c'était le papou de Tristan. [...] Il n'y a pas très longtemps (il a 6 ans), il nous a dit, en fait, moi j'ai deux papas, il y en a un que j'appelle papa, l'autre que j'appelle papou. [...] Nous nous sommes rendu compte, par l'expérience, qu'il n'est pas absolument nécessaire pour un enfant de nommer différemment ses parents. [...] Les lesbiennes sont bien plus en avance là-dessus parce que beaucoup maintenant se font appeler maman toutes les deux et ça ne pose pas de problème. »

\section{LLa gestatrice et la donneuse d'orocyte: deux mères, une mère, pas de mère?}

Les pères gays rencontrés sont traversés par les représentations actuelles de la famille et de la paternité. Obligés d'innover, ils hésitent : ils passent d'une représentation généalogique de la parenté dans laquelle un enfant ne peut avoir qu'un père et une mère à une représentation plus centrée sur la parentalité, permettant d'envisager deux pères sans mère. Ces représentations ne s'excluent pas mutuellement et les enquêtés peuvent les combiner. Ils peuvent par exemple expliquer que leur enfant a deux pères, parce qu'ils sont les parents au quotidien, et une ou deux mères, parce que ces femmes ont contribué à sa venue au monde.

Il y a une diversité de discours quant au statut et à la désignation des femmes qui les ont fait pères. Les hommes rencontrés ont eu recours à la GPA aux États-Unis. Sauf exception, deux femmes ont contribué à la naissance de l'enfant. Une femme apporte son matériel génétique, c'est la donneuse d'ovocyte. Une autre femme porte l'enfant conçu avec le sperme de l'un des deux hommes et l'ovocyte de la donneuse. Ils nomment celle qui a porté « la maman », « la mère porteuse », « la porteuse », « la gestatrice », « la surrogate », ou bien utilisent son prénom. Celle qui a donné ses ovocytes est désignée par les termes de « mère donneuse », « donneuse » ou par son prénom si elle n'est pas anonyme ${ }^{[17]}$. Les appellations traduisent l'ambiguité de ce qu'on entend par le terme «mère » : est-on une mère quand on donne la vie à un enfant sans l'élever ? Il y a ceux qui estiment qu'il y n'y a ni mère, ni maman. Ils considèrent qu'une mère est avant tout quelqu'un qui n'a pas seulement mis au monde un enfant, mais s'implique au quotidien dans les soins et l'éducation. Quelle que soit la manière de la nommer, la place de la femme qui les a aidés à devenir pères n'est jamais vide. Elle a un visage et un nom. Que ces femmes ne soient pas des mères n'empêche pas que des relations s'établissent entre elles ou l'une d'elles, plus généralement, la gestatrice, et le couple d'hommes. Ces relations ne sont pas limitées au temps de la gestation, elles perdurent et instaurent parfois un lien qualifié de familial par les pères et même par les gestatrices. Le recours à la gestation pour autrui n'est pas comparable au recours anonyme à un don de sperme.

[17] Plusieurs pères rencontrés, désireux de pouvoir répondre à la demande éventuelle de leurs enfants quant à leurs origines, ont indiqué avoir demandé à l'agence américaine que la donneuse ne soit pas anonyme. 
Damien et Christophe dont le petit garçon a trois mois, sont très proches de la gestatrice, de son mari et de leurs enfants. Il y a des photos partout dans leur appartement. Ses filles appellent l'enfant leur « surrogacy brother ${ }^{[18]}$. Ils appellent la gestatrice par son prénom et expliquent qu'elle « est la mère puisqu'elle lui a donné naissance, mais qu'en même temps ce n'est pas la mère parce qu'elle ne va pas l'élever. Elle lui a donné naissance, elle l'a porté et elle sera toujours là... ».

Trois paramètres agissent sur les appellations utilisées pour désigner les femmes qui ont aidé ces hommes à devenir pères. Tout d'abord, n'avoir recours qu'à une seule femme ou à deux, influe sur les termes utilisés. Lorsque la même femme a donné son ovule et a porté l'enfant, elle est souvent désignée par un terme maternel : maman, mère, mère biologique, mère de naissance. C'est la mère de leur enfant. Ensuite, l'importance accordée au lien biologique contribue à la désignation de la donneuse. Elle pourra chez certains être la « vraie » mère ou la mère biologique ou la mère donneuse, tandis que chez d'autres, elle sera la donneuse sans connotation maternelle. La plupart des hommes interrogés avaient eu recours à la GPA aux États-Unis et avaient tissé un lien très fort avec la gestatrice. Ils sont parfois non seulement proches d'elle, mais également de son mari et de leurs enfants qui appellent le nouveau-né leur « frère (ou sœur) de surrogacy ». Anne Cadoret ${ }^{[19]}$ et Geneviève Delaisi ${ }^{[20]}$ avaient déjà remarqué cette dynamique relationnelle intense entre les pères gays et les gestatrices. Lorsqu'un couple hétérosexuel a recours à une GPA, une relation très proche, presque fusionnelle peut naitre entre la gestatrice et la mère intentionnelle le temps de la gestation ${ }^{[21]}$. Il est rare que les liens soient maintenus après la naissance avec autant d'intensité. Chez les couples d'hommes, aucune mère intentionnelle ne vient occuper la place de mère qui peut ainsi être préservée pour la gestatrice. Ely Teman ${ }^{[22]}$ et Shireen Kashmeri ${ }^{[23]}$ rapportent que certaines femmes disent préférer porter un enfant pour un couple d'hommes parce que la relation avec eux est moins stressante.

Considérer que les femmes qui ont contribué à la mise au monde de leur enfant sont des mères et maintenir avec ces femmes d'étroites relations ne semble pas avoir d'impact sur la manière dont les couples d'hommes se représentent leur paternité. Excepté ceux, peu nombreux, qui accordent une certaine importance à l'enfantement dans leur représentation de ce que sont un père et une mère, tous les autres disaient être deux pères parce qu'ils plaçaient la paternité dans le registre du lien affectif et du paternage.

\section{$\checkmark$ Considérations finales}

En écoutant ces hommes, il paraît évident que leurs discours à propos des femmes qui les ont aidés diffèrent de ceux que nous avons pu entendre lors des enquêtes menées auprès des mères

[18] Frère de GPA.

[19] Anne CADORET, « L'homoparentalité, construction d'une nouvelle figure familiale », Anthropologie et sociétés, $\mathrm{n}^{\circ}$ 24/3, 2000, pp. 39-52 ; Anne CADORET, Des parents comme les autres, Paris, Odile Jacob, 2002.

[20] Geneviève DELAISI de PARCEVAL, Famille à tout prix, Seuil, 2008.

[21] Ely TEMAN, Birthing a mother. The surrogate body and the pregnant self, University of California Press, 2010.

[22] Ibid. p. 131.

[23] Shireen KASHMERI, Unraveling Surrogacy in Ontario, Canada. An Ethnographic Inquiry on the Influence of Canada's Assisted Human Reproduction Act (2004), Surrogacy Contracts, Parentage Laws, and Gay Fatherhood, Montreal, Quebec, Concordia University, 2008. 
lesbiennes à propos des donneurs. Celles-ci opèrent facilement une disjonction entre père et géniteur, notamment lorsque ce dernier n'est pas connu. Les pères gays distinguent moins aisément mère et gestatrice. Les mères lesbiennes dont le projet parental était un projet de couple et qui se font appeler toutes les deux mamans, ne les désignent pratiquement jamais par un terme issu du lexique de la parenté. C'est un géniteur, un donneur parce qu'un père, selon elles, est quelqu'un qui s'implique auprès de son enfant. De leur côté, plusieurs pères gays qui ont eu recours à la GPA désignent la femme porteuse par un terme maternel, la mère, la mère porteuse, la mère de naissance... et quelques-uns l'appellent mère biologique ou donneuse. Pourquoi les hommes semblent-ils adhérer davantage à la représentation dans laquelle procréation et parenté ont partie liée ? Pourquoi les pères gays et les mères lesbiennes évoquent-ils ces « tiers de procréation » de manière différente ? Il est possible que se représenter le géniteur comme un père porte atteinte à leur vision conjugale. Peut-on faire un enfant avec un homme sans vivre avec lui une sorte de relation conjugale?

L'implication corporelle du don de sperme est minimale comparée à celle des donneuses d'ovocyte ou des gestatrices. Une fois la conception réalisée, la présence et l'implication d'un homme n'a rien d'obligatoire pour faire d'une femme une mère. Ce qui n'est pas le cas des hommes qui, pour leur part, doivent passer par les femmes pour devenir pères. Dans le droit, en dehors de l'adoption, on ne peut devenir père sans nommer celle qui a accouché de l'enfant. Les nommer, voire être en relation avec ces femmes, ne serait pas menaçant puisque c'est la condition de leur paternité.

Comment les donneurs de sperme, les donneuses d'ovocyte et les gestatrices se situent-ils par rapport aux enfants nés de leur don ? René Almeling, dans son étude ${ }^{[24]}$ sur les tiers de procréation aux États-Unis, montre que les donneurs de sperme, même s'ils ne sont liés par aucun droit ni obligation envers les enfants nés de leur don, vivent ses enfants comme les leurs et se vivent comme des pères. En revanche, les donneuses d'ovocytes et les gestatrices clament haut et fort qu'elles ne sont pas du tout des mères et qu'elles ne font que contribuer à donner la vie. Contrairement aux hommes qui se déclarent pères dès qu'ils savent avoir donné la vie, sans se sentir obligés de s'impliquer davantage, les femmes ne peuvent se dire mères sans être automatiquement assignées aux tâches maternelles, sauf à accepter de passer pour « mauvaise mère ». Pour échapper à cette qualification, et à la culpabilité d'abandonner un enfant, elles ne peuvent que revendiquer de ne pas être une mère, même si elles ont contribué à le mettre au monde. Cela leur est facilité par la scission de la maternité en trois liens distincts, génétique, gestationnel et affectif. N'être concernées que par la gestation ou le don d'ovule leur permet de ne pas devenir mères des enfants auxquels elles ont donné la vie.

\section{¿L'explication est-elle à chercher du côtéd'un rapport au corps?}

Du côté masculin, les tiers, gestatrice ou donneuse, sont des personnes dont l'existence est réelle et concrète mais dont la contribution reste extérieure au corps des hommes, qui peuvent sans risque la nommer, voire installer une relation dans la durée et dans la réciprocité. Ils peuvent

[24] René ALMELING, Sex cells: the medical market for eggs and sperm, Berkely and Los Angeles, California, University of California Press, 2011. 
la qualifier de mère, le risque n'est pas grand puisqu'elle revendique ne pas en être une. Tandis que du côté féminin, envisager une relation concrète avec un donneur non anonyme, qui pourrait éventuellement se sentir père et dont le sperme a été introduit dans le corps de l'une des deux femmes pourrait sembler à certaines fragilisant pour l'intégrité du couple de femmes. Alain Ducousso-Lacaze ${ }^{[25]}$ met ainsi en évidence l'existence de fantasmes hétérosexuels chez certains couples de mères lesbiennes, vis-à-vis du donneur.

Les couples hétérosexuels, s'ils maintiennent des liens avec la gestatrice n'ont généralement aucune information identifiante sur la donneuse d'ovocyte à moins qu'elle ne soit la mère d'intention. La plupart des hommes rencontrés dans cette enquête font non seulement en sorte que l'enfant puisse accéder à l'identité des personnes qui ont permis sa venue au monde, mais également qu'une rencontre soit possible si l'enfant en exprime le besoin. Sont-ils moins menacés par la connaissance de l'identité des donneuses parce que leur infertilité fonctionnelle ne les blesse pas comme peut le faire l'infertilité pathologique dont souffrent les couples hétérosexuels ? Les pères gays sont-ils plus préoccupés d'une éventuelle quête des origines chez leurs enfants ? Sont-ils plus motivés à restituer un récit de sa venue au monde le plus authentique possible pour inscrire leurs enfants dans une commune humanité ? Ce sont là des hypothèses vraisemblables d'autant qu'ils ne sont jamais tentés de passer pour avoir procréé ensemble.

Une étude israélienne ${ }^{[26]}$ révèle que le recours à un don de gamète homologue est aussi difficile à accepter par les femmes que par les hommes. Un don de sperme pour un couple hétérosexuel menace probablement davantage le père intentionnel que la mère. Réciproquement, une mère intentionnelle craint probablement davantage que le père, une mise en cause de son lien parental en cas de recours à un don d'ovocyte. Autrement dit, l'articulation des liens biologiques et sociaux élaborée par les pères gays diffère sensiblement de celle des couples hétérosexuels infertiles. En effet, d'une part, le don de gestation et le don d'ovocyte qui contribuent à faire d'un couple d'hommes deux pères ne sont pas des dons homologues. De sorte qu'aucun des deux n'est menacé par le don de gestation ou le don d'ovocyte. D'autre part, ces dons n'ont pas pour effet de faire passer le couple pour un couple procréateur. Au contraire, pour se représenter leur double paternité, les couples d'hommes doivent valoriser la dimension affective et sociale de celui qui n'est ni père biologique ni père légal. Pour ce faire, ils vont parfois jusqu'à occulter ou ignorer délibérément le caractère biologique du lien afin d'éviter de créer une asymétrie trop importante entre eux et dans le regard d'autrui. Les couples de femmes de leur côté ne peuvent user de cette stratégie, avoir porté un enfant ne peut s'occulter aussi facilement. Elles doivent, si elles veulent être deux mères et donner de l'importance à la maternité élective, valoriser la dimension conjugale de leur projet parental que la présence d'un père au quotidien ou seulement dans le discours risque de fragiliser.

[25] Alain DUCOUSSO-LACAZE, Propos du père dans la parentalité lesbienne, Divan Familial, $\mathrm{n}^{\circ}$ 13, 2004, pp. 29-42.

[26] Ruth LANDAU, Ruth WEISSENBERG, Igael MADGAR, " A child of "hers" : older single mothers and their children conceived through IVF with both eggs and sperm donation", Fertil Steril, n 90/3, 2008, pp. 576-583. 